\title{
Smartphone Addiction and Its Relationship with Psychological Health among Clinical Years Medical Students of International Islamic University Malaysia (IIUM), Kuantan
}

Azwanis Abdul Hadi, Hawari Musyir Mohd Nawawi, Nurafifah Shamsuri, Nurul Najihah Rahim, Hafizah Pasi

Department of Family Medicine, International Islamic University Malaysia, Kuantan

Introduction: Smartphones has now become a common and almost essential tool in our daily living activities. Students are among those who uses smartphones regularly which could lead to addiction if overused. This study aims to measure the prevalence of smartphone addiction and its relationship with psychological health among clinical year medical students in International Islamic University Malaysia (IIUM), Kuantan. Materials and method: A total of 203 Medical students in their clinical years were recruited via quota sampling method. The students filled in a four part online-based questionnaire consisted of questions on sociodemographic characteristics, smartphone usage information, Smartphone addiction scale-short version (SAS-SV) and WHOQOL-BREF. Data was analysed using IBM SPSS Statistics. Result: The prevalence of smartphone addiction among clinical year Medical students in IIUM was 51\%. Bivariate analysis showed that majority of students chose social networking as their primary purpose of smartphone usage and this is significantly associated with smartphone addiction ( $p$-value:0.037). Following multiple logistic regression analysis, students who had chosen games as their primary purpose of smartphone usage are $88 \%$ less likely to be related with smartphone addiction ( $\mathrm{p}$-value: $0.009,95 \% \mathrm{Cl}: 0.025-0.595)$ compared to those who used it for academic performance tasks. Students who are not addicted to smartphones reported significantly better psychological quality of life compared to those who are addicted ( $p$-value<0.001). Conclusion: Half of IIUM's Medical students in clinical years have smartphone addiction and those with smartphone addiction was found to have poor psychological health. Immediate intervention such as creating awareness in the campus is important in curbing this problem. 